\title{
Nonconventional optical systems using varifocal lenses
}

\author{
J. Ojeda-Castaneda, ${ }^{* 1}$ and Cristina M. Gómez-Sarabia ${ }^{2}$ \\ ${ }^{1}$ Electronics Department, Campus Salamanca, University of Guanajuato, 36885 Guanajuato, México \\ ${ }^{2}$ Digital Arts, Campus Salamanca, University of Guanajuato, 36885 Guanajuato, México
}

Received March 7, 2015; accepted March 31, 2015; published March 31, 2015

\begin{abstract}
We discuss the first order design of four optical devices, which use varifocal lenses for implementing the following optical systems: a single-lens zoom system, two-lens afocal system with tunable angular magnification, three-lens telephoto objective with an extremely low, tunable telephoto ratio (which does not require any axial displacement between its composing elements) and a telephoto objective that has a zero Petzval sum.
\end{abstract}

Several authors have proposed different techniques for implementing varifocal lenses. Some proposals follow the method that goes back to Kitajima [1-3]. Improvements to this proposal were presented independently and simultaneously by Lohmann [4-7] and by Alvarez [8-10]. This later method has inspired the use of two zone plates for generating varifocal Moiré fringes [11-14].

For implementing varifocal lenses, other authors have explored the use of liquid lenses [15, 16], and some others employ transparent membranes that can be acoustically controlled [17, 18]. Recently, based on the proposals in reference 7 , we have suggested the use of vortex pairs for obtaining novel, tuneable focalizers [19, 20].

Related to the above research trends, it has been noted that varifocal lenses are useful for setting nonconventional telephoto objectives [21], as well as optical zoom systems [22-24].

Our aim here is to revisit the Gaussian optics design of the four optical systems that use varifocal lenses: a) a single-lens zoom system; b) two-lens afocal system with tunable angular magnification; c) three-lens telephoto objective that does not require any axial displacement between its composing elements, and that can be used for obtaining extremely low, tuneable magnifications; and d) improved version of the previous three-lens telephoto objective, which now has a zero Petzval sum.

As depicted in Fig. 1a), we indicate that by axially displacing a varifocal lens between a pair of conjugate planes, one can obtain the desired magnification. Then, at this specific position, one selects the optical power that is needed for mapping the input plane into the output plane. These planes are separated by the fixed distance $\mathrm{T}$, which

\footnotetext{
"E-mail: jojedacas@ugto.mx
}

is here denoted as the throw [25].

a)

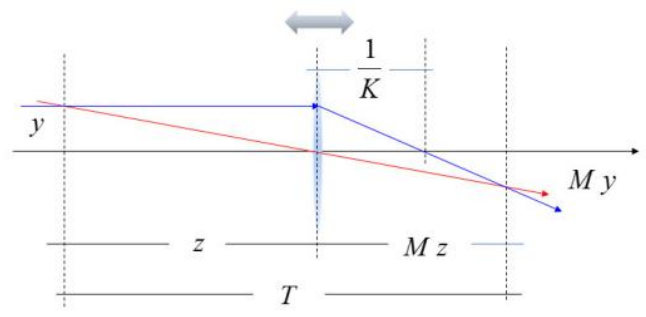

b)

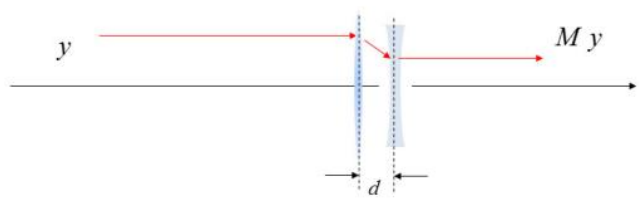

Fig. 1. Schematics of two optical systems using varifocal lenses.

In mathematical terms, if the desired magnification is $M$, then the distance from the lens to the input plane is

$$
z=\frac{T}{M-1}
$$

And the needed optical power is

$$
K=-\frac{(1-M)^{2}}{M T}
$$

The main advantage of this design is its simplicity. Didactically speaking, this example shows the usefulness of a varifocal lens. Next, we describe an afocal device with tunable angular magnification.

As depicted in Fig. 1b), we employ now two composing elements that are separated by fixed distance, $d$; such that

$$
d=\frac{1}{K_{1}}+\frac{1}{K_{2}} .
$$

Next, we note that from the first lens to the second lens, 
the lateral magnification is

$$
M=\frac{y_{2}}{y_{1}}=-\frac{K_{1}}{K_{2}}
$$

Of course, the angular magnification is the inverse of Eq. (4). Now, by combining Eqs. (3) and (4), we obtain the following results. For continuously varying the magnification of this afocal device, one requires to use a first varifocal lens, whose optical power changes as

$$
K_{1}=\frac{1-M}{d}
$$

The second element is also a varifocal lens, whose optical power changes as

$$
K_{2}=-\frac{1-M}{M d}
$$

Hence, even when the separation between the two varifocal lenses is constant, the afocal system can change continuously its angular magnification.

In what follows, we show that the previously described afocal device can be transformed into a telephoto objective with the following features: the magnification is tunable, the system does not require any axial displacement, and one can obtain extremely low telephoto ratios.

a)

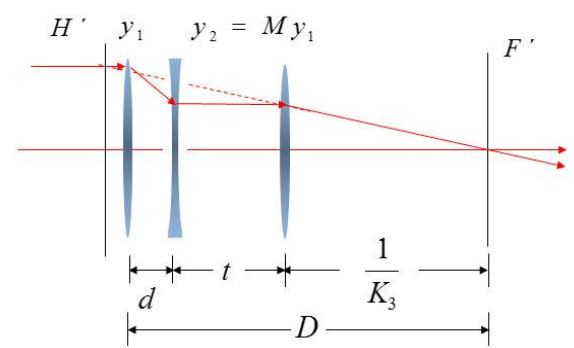

b)

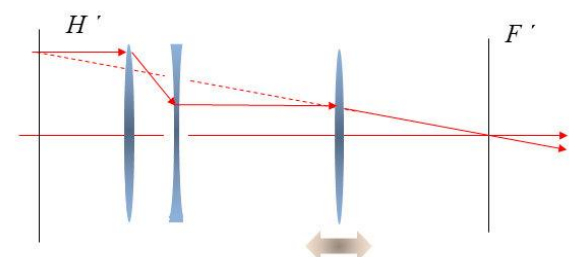

Fig. 2. Schematics of two types of telephoto objectives.

From Fig. 2a) we note that the initial paraxial ray crosses the optical axis at the back focal plane of the third lens; that is the back focal length is $f_{\text {back }}=1 / K_{3}$. Furthermore, by paraxial ray tracing, it is possible to show that the equivalent optical power is

$$
K=\frac{1}{f^{\prime}}=M K_{3} .
$$

In Eq. (7), we denote as $f^{\prime}$ the equivalent focal length. If one uses Cox definition of telephoto ratio [26], this design has the following telephoto ratio

$$
\mathfrak{R}=\frac{f_{\text {back }}}{f^{\prime}}=\frac{K}{K_{3}}=M .
$$

It is apparent from Eq. (8) that by using this device, one can obtain extremely low values of telephoto ratios. In addition, from Fig. 2, we note that for this design the separation between the second lens and the third lens, say $t$, can be properly selected for fulfilling the possible constrain of a device with maximum length $D$.

$$
t=D-\left(d+\frac{1}{K_{3}}\right) .
$$

In what follows we show that this optical device can have a zero Petzval sum.

In the previous design, we fixed the value of $K_{3}$, then by using Eqs. (5) and (6), we have that the Petzval sum is different from zero

$$
\sum_{s=1}^{3} K_{s}=-\frac{(1-M)^{2}}{M d}+K_{3} .
$$

However, one can obtain a similar optical system that has zero Petzval sum. Trivially, the third lens has to be a varifocal lens, whose optical power changes as

$$
K_{3}=\frac{(1-M)^{2}}{M d} .
$$

Since now the third lens is a varifocal lens, then one must allow for an axial displacement of the third element. By paraxial ray tracing, one can show that the equivalent optical power is now

$$
K=\frac{(1-M)^{2}}{d}
$$

From Eqs. (5) and (12) we obtain that 


$$
K=(1-M) \mathrm{K}_{1} .
$$

In Figure 3 we plot the variations of $\mathrm{K}_{1}, \mathrm{~K}_{2}, \mathrm{~K}_{3}$ and $\mathrm{K}$ as functions of the magnification $\mathrm{M}$, in the range $0.2 \leq \mathrm{M} \leq$ 1. It is apparent from Fig. 3 that the equivalent optical power is indeed proportional to the optical power of the first lens: as indicated in Eq. (13). Thus, we claim that the afocal device in Fig. 1b) can be transformed into a threelens telephoto objective with the following features: the magnification is tunable, one can obtain extremely low telephoto ratios, and the system has a zero Petzval sum.

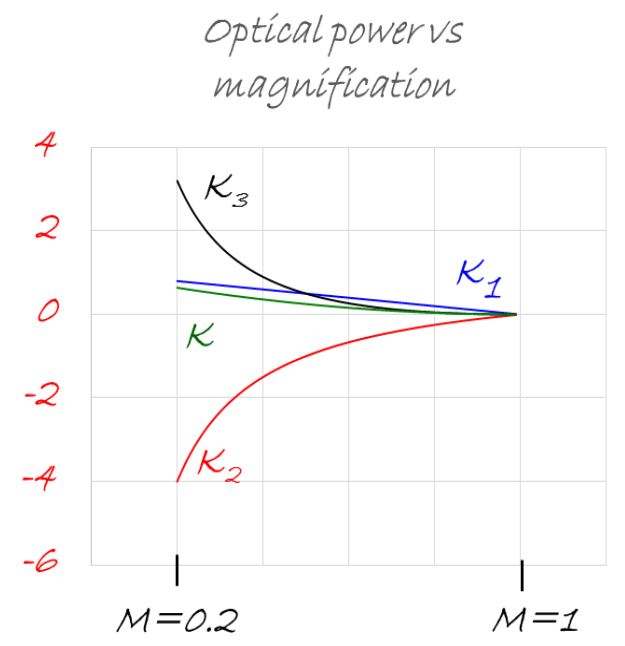

Fig. 3. Optical power as a function of lateral magnification.

The main disadvantage is that one requires an axial displacement of the third component. In the following table we summarize the main characteristics of the previous proposals.

Table 1. Basic features of the proposed designs

\begin{tabular}{|c|c|c|c|c|}
\hline & $\begin{array}{l}\text { Physical } \\
\text { constrain }\end{array}$ & Afocal & $\begin{array}{c}\text { Axial } \\
\text { displacement }\end{array}$ & $\begin{array}{l}\text { Petzval } \\
\text { sum }=0\end{array}$ \\
\hline $\begin{array}{l}\text { Single } \\
\text { lens }\end{array}$ & $T$ & No & Yes & No \\
\hline $\begin{array}{l}\text { Two } \\
\text { lenses }\end{array}$ & $d$ & Yes & No & No \\
\hline $\begin{array}{l}\text { Three } \\
\text { lenses }\end{array}$ & $d$ and $D$ & No & No & $\mathrm{No}$ \\
\hline $\begin{array}{l}\text { Three } \\
\text { lenses }\end{array}$ & $d$ and $D$ & No & Yes & Yes \\
\hline
\end{tabular}

In conclusion, we have explored the possibilities of using a single varifocal lens for obtaining a zoom system, which works between two fixed conjugate planes. Next, we have described an afocal device that employs two varifocal lenses, which are separated by a fixed distance.
By using this telescopic device, one can tune continuously the angular magnification.

The afocal device was transformed in a telephoto objective, by using a third lens. We have shown that the telephoto objective has a telephoto ratio that is in inverse proportion to the angular magnification. Thus, one can obtain extremely low telephoto ratios. However, this design does not have a zero Petzval sum. Finally, we have shown that by allowing axial displacements of a third varifocal lens, one can achieve a zero Petzval sum.

We gratefully acknowledge the financial support of CoNaCyT, research project 157673, CA40120112. We thank the reviewers for their useful suggestions.

\section{References}

[1] I. Kitajima, British Patent 250, 268 (July 29, 1926).

[2] W.T. Plummer, J.G. Baker, J. van Tassell, Appl. Opt. 38, 3572 (1999).

[3] W.T. Plummer, Appl. Optics 21, 196 (1982).

[4] A.W. Lohmann, British Patent 998, 191 (May 29, 1964).

[5] A.W. Lohmann, Republic Française 1, 398, 351 (June 10, 1964).

[6] A.W. Lohmann, Italy Patent 727, 848 (June 19, 1964).

[7] A.W Lohmann, Appl. Opt. 9, 1669 (1970).

[8] L.W. Alvarez, U.S. patent 3,305,294 (21 February 1967).

[9] L.W. Alvarez , W. E. Humphrey, U.S. patent 3,507,565 (21 April 1970).

[10] L.W. Alvarez, J. Am. Optometric Assoc. 49, 24 (1978).

[11] A. Lohmann, D.P. Paris, Appl. Opt. 6, 1567 (1967).

[12] G.L. Rogers, L.C.G. Rogers, Opt. Acta 24, 15 (1977).

[13] J.M. Burch, D.C. Williams, Appl. Opt. 16, 2445 (1977).

[14] A. Kołodziejczyk, Z. Jaroszewicz, Appl. Opt. 32, 4317 (1993).

[15] H.W. Ren, Y.H. Fan, S. Gauza, S.T. Wu, Appl. Phys. Lett. 84, 4789 (2004)

[16] S. Reichelt, H. Zappe, Opt. Expr. 15, 14146 (2007).

[17] A. Mermillod-Blondin, E. McLeod, C.B. Arnold, Opt. Lett. 33, 2146 (2008).

[18] M. Duocastella, G. Vicidomini, A. Diaspro, Opt. Exp. 22, 19293 (2014).

[19] J. Ojeda-Castañeda, C.M. Gómez-Sarabia, S. Ledesma, Proc. SPIE 8833, 883306 (2013).

[20] J. Ojeda-Castaneda, S. Ledesma, CM. Gómez-Sarabia, Phot. Lett. Poland 5, 20 (2013).

[21] J. Schwiegerling, C. Paleta-Toxqui, Appl. Opt. 48, 1932 (2009).

[22] J.-H. Sun, B.-R. Hsueh, Y.-C. Fang, J. MacDonald, C.-C. Hu, Appl. Opt. 48, 1741 (2009).

[23] J. Ojeda-Castaneda, C.M. Gómez-Sarabia, S. Ledesma, Asian J. Phys. 23, 415 (2014)

[24] J. Ojeda-Castaneda, C.M. Gómez-Sarabia, S. Ledesma, Asian J. Phys. 23, 535 (2014).

[25] P. Mouroulis, J. Macdonald, Geometrical Optics and Optical Design (New York, Oxford University Press, 1997).

[26] A. Cox, Photographic Optics (London, Focal Press, 1974). 